$\stackrel{W}{=}$

Global journals Inc.

है

\title{
Controversial Issues of Clinical Immunology. Modern Concepts about the Pathogenesis of Infections
}

\author{
By Zemskov, V. M., Zemskov, A. M., Pronko, K. N., Afanasiev, S. S., Zemskova, V. A. \\ \& Revishvili, A. Sh. \\ Voronezh State Medical University
}

Abstract- It is postulated that a suppressed or stimulated state of immune reactivity plays an important role in the induction of an infection process, which triggers the activation of microbiota pathogens. Infection-driven destruction of cells of a macroorganism promotes the release of endogenous low molecular weight nucleic acids, mostly RNA, which, within the first hours/days, are responsible for the stimulation of replication and toxin formation by microflora with subsequent disease exacerbation, and as a result, in a few days, cause an increase in antigenicity, immunogenicity, antibiotic susceptibility of pathogens, and activation of nonspecific and specific resistance of the body, with the resulting immune activation. Concurrently with these processes, the intensification of free-radical lipid and protein oxidation is observed, with the formation of immunosuppressive compounds which are neutralized by enzymatic and nonenzymatic factors of the antioxidant system. Thus, in infection processes homeostasis is achieved by means of a sequential cascade of microbial, immune, and metabolic mechanisms which induce, stimulate, and inhibit the responsiveness of the body.

Keywords: microbiota, hospital-acquired infection, antioxidants, metabolic processes, low molecular weight RNA.

GJMR-C Classification: NLMC Code: QW 180

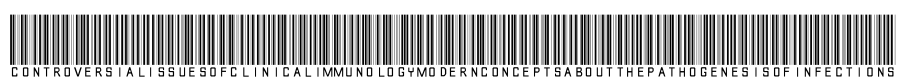

Strictly as per the compliance and regulations of:

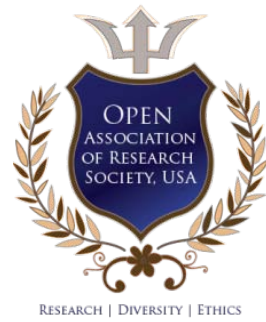

(c) 2019. Zemskov, V. M., Zemskov, A. M., Pronko, K. N., Afanasiev, S. S., Zemskova, V. A. \& Revishvili, A. Sh. This is a research/review paper, distributed under the terms of the Creative Commons Attribution-Noncommercial 3.0 Unported License http://creativecommons.org/licenses/by-nc/3.0/), permitting all non-commercial use, distribution, and reproduction in any medium, provided the original work is properly cited. 


\title{
Controversial Issues of Clinical Immunology. Modern Concepts about the Pathogenesis of Infections
}

\author{
Zemskov, V. M. ${ }^{\alpha}$, Zemskov, A. M. ${ }^{\sigma}$, Pronko, K. N. ${ }^{\circ}$, Afanasiev, S. S. ${ }^{\omega}$, Zemskova, V. A. ${ }^{*}$ \\ \& Revishvili, A. Sh. ${ }^{\S}$
}

Abstract-It is postulated that a suppressed or stimulated state of immune reactivity plays an important role in the induction of an infection process, which triggers the activation of microbiota pathogens. Infection-driven destruction of cells of a macroorganism promotes the release of endogenous low molecular weight nucleic acids, mostly RNA, which, within the first hours/days, are responsible for the stimulation of replication and toxin formation by microflora with subsequent disease exacerbation, and as a result, in a few days, cause an increase in antigenicity, immunogenicity, antibiotic susceptibility of pathogens, and activation of nonspecific and specific resistance of the body, with the resulting immune activation. Concurrently with these processes, the intensification of free-radical lipid and protein oxidation is observed, with the formation of immunosuppressive compounds which are neutralized by enzymatic and nonenzymatic factors of the antioxidant system. Thus, in infection processes homeostasis is achieved by means of a sequential cascade of microbial, immune, and metabolic mechanisms which induce, stimulate, and inhibit the responsiveness of the body.

Keywords: microbiota, hospital-acquired infection, antioxidants, metabolic processes, low molecular weight RNA.

\section{INTRODUCTION}

T he modern trend for increase in infectious morbidity, with modifications of the characteristic features of infections, including immune ones, is indicative of a distortion of population resistance and low efficacy of diagnostic and treatment techniques

Author $\alpha$ : Professor of allergology and immunology, PhD, MD, Chief of Clinical Immunology Group AV Vishnevski National Medical Research Center of Surgery, Moscow, Russia. e-mail: arturrego@yandex.ru

Author $\alpha$ : Professor of allergology and immunology, PhD, MD, Head of Research and Development at Facecontrol, Inc., Florida, Miami, USA.

Author o: Professor of allergology and immunology, PhD, MD, Chief of Department of Microbiology, Burdenko Voronezh State Medical University, Voronezh, Russia.

Author $\rho$ : Doctor of clinical psychology, Vice-President at Facecontrol, Inc., Florida, Miami, USA.

Author $\omega$ : Professor of allergology and immunology, PhD, MD, Chief Researcher of G. N. Gabrichevsky Research Institute for Epidemiology and Microbiology, Moscow, Russia.

Author ¥: Assistant professor of Department of Pathophysiology of Burdenko, Voronezh State Medical University, Voronezh, Russia.

Author \&: Academician of RAS, Professor of cardiac surgery, PhD, MD, Director AV Vishnevski National Medical Research Center of Surgery, Moscow, Russia.
(Vorobyev et al., 2010; Pokrovsky et al., 2013; Zemskov AM and Zemskov VM, 2016; Pokrovsky, 2012).

A concept of the "main components of the pathogenesis of infections," i.e. immune reactivitymicrobiota-nucleic acids-metabolic processes, has been developed based on the analysis of modern data.

a) Interactions between Immune Reactivity and Microbiota during Infection Processes

i. Immune Reactivity

Immune reactivity is determined by the number and activity of lymphoid phagocytic cells observed in circulating blood and locally in tissues, the state of the complement system, nonspecific resistance parameters, killer cells, immune globulins, specific antibodies, cytokines, and other parameters in a given patient at the present moment (Zemskov et al., 2016a).

\section{ii. Colonization Resistance}

Immune reactivity is contiguous with the phenomenon of colonization resistance of mucosa of exposed body cavities, i.e. resident microflora, the composition of which is determined by microbial adhesion inhibitors, biocidal and biostatic secretory products, mechanical factors (ciliated epithelium, integrity of the skin and mucosa, mucociliary epithelium movement, bowel motility, mucosal cell desquamation), antimicrobial effects of salivary secretion, bile, gastrointestinal contents, the composition and the amount of mucin, oxygen tension through the thickness of biofilm, $\mathrm{pH}$ of the medium, and by environmental factors (Karaulov et al., 2017).

\section{iii. Microbiota (microbiocenosis)}

It includes bacteria, spirochetes, bacteriophages, mycoplasma, chlamydia, rickettsia, viruses, prions, and other microorganisms on the surface of the skin and mucosa of exposed cavities. Diseases of microbiota (dysbiosis) are disturbances of the balance between representatives of normal microflora of the body characterized by significant abnormal quantitative and qualitative composition of microbiocenosis. According to recent data, anaerobes account for $90 \%$ of normal intestinal microflora, thus they cannot grow on conventional media. In other words, what is known as dysbiosis accounts for $10 \%$ of the existing reality (Zemskov et al., 2016a). 
There are 4 stages of dysbiosis: normocenosis (I), intermediate (II), dysbiosis (III), and significant inflammation (IV). It is generally assumed that stage I or II dysbiosis is observed in healthy patients, and stage III or IV dysbiosis-in patients with premonitory symptom and favorable course of the infection. In case of recovery and eradication of the pathogen, a reversion to stage I or II takes place, while in cases of chronic diseases stage III or IV dysbiosis is observed for a longer period of time (Karaulov et al., 2017).

There are several possible associations between the immune reactivity level in patients and their microbiota.

1. In healthy individuals, there is a balance between microbiota mostly represented by commensals (despite the lack of clear delineation between them and opportunistic pathogens) and immune reactivity.

2. In immune compromised patients (AIDS), slightly virulent and even saprophytic microflora of the skin and mucosa may become capable of causing infectious disorders.

3. In case of immune reactivity activation due to the accumulation of antibacterial factors, such as lysozyme, lactoferrin, stimulated neutrophils, macrophages, immune globulins, etc., in the epithelium, microorganisms respond with the activation of aggression enzymes, such as neuraminidase and hyaluronidase, release of endotoxins, etc., with a certain probability of promoting infections.

4. In some cases, pathological conditions due to highly virulent pathogens develop irrespective of a patient's immune status and microbiocenosis.

5. These processes are controlled by built-in pattern recognition receptors (PRRs), which are conservative molecular structures only found in microorganisms (pathogen associated molecular patterns (PAMPs)) and which control cytokine cascade, inflammatory reactions, immunoglobulin production, etc. There are several PRR families: TOLL-like receptors, NOD-like receptors, mannose lectin and scavenger receptors, and complement system factor receptors. For example, the activation of mucosal TOLL-like receptors (TLR-4) regulates resistance against gram-negative pathogens, TLR2-against gram-positive pathogens, and TLR-3 and TLR-8-against viral pathogens (Karaulov et al., 2017).

b) The Role of Nucleic Acids in Immune Response, Infections, and Metabolic Processes

Development of any pathological processes and a number of physiological ones is accompanied by destruction of cells of various tissues of the body caused by microorganisms, their toxins, aggression enzymes, etc., which results in enrichment of the internal environment with fragments of low molecular weight nucleic acids and factors promoting their release. This is confirmed by published data, according to which significant changes in plasma concentrations of nucleic acids, primarily RNAs, are observed during various infectious diseases. They include the following: nonspecific pulmonary inflammatory diseases, acute dysentery, viral hepatitis, pyelonephritis, purulent processes, etc. (Zemskov et al., 2016b).

\section{i. Effect on Immune Reactivity}

In model experiments during the administration of low molecular weight RNA in rodents 4-24 hours before inoculation, stimulation of nonspecific protection factors, such as the complement and properdin systems, interferons, $\beta$-lysines, and infectious agent growth inhibitors is observed, together with promotion of mobility, as well as absorbing and metabolic capacity of monocytes/macrophages. At later stages, they are accompanied by the stimulation of specific cellmediated and antibody-mediated immune response. The spectrum of action of ribonucleotides includes quantitative and functional characteristics of the main populations and subpopulations of lymphoid cells, phagocytic activity, processes of stem cell differentiation, cooperation of $\mathrm{T}$ - and B-lymphocytes and macrophages, formation of cytokines, desensitization of the body, immune memory depression, antigen redeposition, expression of various cell receptors, etc. These dynamic processes are differently directed and implement the intimate mechanism of immune system homeostasis regulation.

The following biological phenomena were observed in model experiments and clinical observations when using sodium nucleinate (sodium salt of low molecular weight yeast RNA), an equivalent of ribonucleotides naturally released during infection processes (Zemskov et al., 2013).

\section{a. Antiviral Action}

Antiviral action is mediated by activation of $\mathrm{T}$ - and B-cell dependent immune responses, phagocytosis, interferon formation, and other mechanisms.

\section{b. Detoxication}

Detoxication is ensured by the normalization of nucleic-protein synthesis, stimulation of phagocytosis, reparative processes in the liver, an increase in the activity of several enzymes, etc.

\section{c. Adjuvanticity}

Adjuvanticity is implemented through potentiation of cell-mediated and antibody-mediated immune responses, phagocytic component, activation of stem cell and T-helper migration, cooperation of main cell participants of immune response, RNA and antigen complex formation which leads to a rapid increase in immunogenicity of the antigen. This also boosts the 
primary and secondary immune responses to thymusdependent and thymus-independent antigens, corpuscular, chemical, mixed, and live vaccines, as well as anatoxins.

\section{d. Revaccination Effect}

The revaccination effect is induced as a result of increased interaction between $\mathrm{T}$ - and B-cells with macrophages, antigen redeposition, and immune memory cell depression.

\section{e. Immune Modulation}

It is based on the pluripotent effect of low molecular weight RNA on metabolic and immune processes.

\section{f. Desensitization}

Desensitization is mediated by modulation of the suppressor component of the immune system, stabilization of membranes and enzyme systems of the respective cells destroying biogenic amines, etc.

c) The Mechanism of Implementation of General Biological Effects of Nucleic Acids

As for the mechanism of implementation of general biological effects of RNA, they are still understudied. According to published data, nucleic acids and, first of all, RNA replenish the supply of substances required for metabolism, thus affecting vital functions of the body in general. Ribonucleotides and their components form a part of many cell metabolism products (ATP, GTP, UTP, CMP), as well as several coenzymes and phospholipids required for normal performance of certain functions. It is noteworthy that ATP is an energy source, cytosine nucleotides are involved in the synthesis of lipids, uridine-in polysaccharide metabolism, and guanine nucleotidesin the synthesis of proteins. Apparently, this mechanism is not the only one or the main one. In any case, W. Braun states the following in the context of microorganisms: "... actual stimulation of cell multiplication turns out to be significantly greater than estimated stimulation based on the assumption that endogenous nucleic acids are only used as building blocks" (Braun, 1968). It is certain that an indirect path of action of nucleic acids on cells exists, probably through a system of cyclic nucleotides. Their effect through the respective nucleotide receptors on the cellular membrane also cannot be ruled out as it was suggested that some receptors for fragments of low molecular weight RNA exist on cell surface and translate its effect. It has been shown that in cases of the effect of healthy individuals' lymphocytes on receptors, purine nucleotides demonstrate a stimulating effect to a greater extent, while pyrimidine nucleotides are mostly characterized by inhibitory effects. AMP demonstrates the highest activity, while GMP has the lowest activity, and a combination of complementary GMP and CMP was highly productive. However, it turned out to be less active than the total "natural" product sodium nucleinate and a complex of four nucleotides. Inhibition of the expression of specific receptors in immature pre-B-cells and their stimulation in pre-T-cells is observed. Minimum changes have been observed for lymphocytes with helper and suppressor functions. At the same time, in patients with acute dysentery, the expression of cell receptors was inhibited to the greatest extent due to the effect of AMP purine, CMP pyrimidine, and a combination of both. All mononucleotides and their combinations had minimum impact on T-suppressors, while GMP + CMP promoted their inhibition. GMP+UMP ensured significant stimulation of the expression of Bcell receptors. There are still no data on the effect of these changes in the receptor apparatus on the properties of lymphocytes, but various biological effects of mononucleotides have been confirmed. CMP and UMP 9-19-fold stimulate antibody-producing cells formation, while AMP decreases their formation 10-fold against the baseline. On the other hand, AMP inhibits antigen-specific reactions and increases delayed hypersensitivity (Zemskov et al., 2016b).

\section{d) Effect on Infection}

Accumulation of low molecular weight ribonucleotides in the body stimulates the growth of microorganisms of various taxonomic groups, selection of their virulent clones, and production of exotoxins early in the course of an infection process, which is accompanied by infection potentiation. This phenomenon was documented in model experiments with administration of a broad spectrum of grampositive, gram-negative, and other pathogens in susceptible animals along with RNA preparations. This caused an obvious worsening of the infection, which was judged based on the decrease in $L_{50}$ values, survival rate, and average life span of rodents, and an increase in skin necrosis area in case of an intracutaneous coinjection of gaseous gangrene pathogen and RNA preparations. Subsequently, the same nucleic acid preparation caused an increase in antigenicity, immunogenicity, specific immune defense factors, which was experimentally reproduced by administration of a nucleic acid stimulator and antigens or vaccine simultaneously or 24-72 $\mathrm{h}$ after the injection immune preparations of laboratory animals. When growth of microorganisms on the solid bacteriological medium was with RNA in vitro - microorganisms received antibiotic susceptibility.

\section{e) Effect on the Metabolic Processes of the Macroorganisms}

The following effects were documented when using low molecular weight RNA in vitro experiments, in vivo experiments, and clinical observations (Zemskolv et al., 1985a; Zemskov V. M. and Zemskov A.M., 1992). The stimulation of RNAs, DNAs, and protein synthesis, the accumulation of ATP and ADP, and the activation 
of monoaminooxidase and $\alpha$-glycerophosphate dehydrogenase was observed in lymphocytes of the spleen; the increased biosynthesis of mitochondrial, nuclear, and cytoplasmic RNAs and the increased activity of tryptophan pyrrolase were observed in the liver. Increased oxygen consumption and decreased glycolysis rate were observed in normal granulocytes. The activation of protein formation was observed in bone marrow and brain cells and in tumor cell cultures, while macrophages were characterized by the activation of glycolysis, oxidative phosphorylation, amino acid catabolism, increased activity of dehydrogenases, mitochondrial $\alpha$-glycerophosphate dehydrogenase, glutamate dehydrogenase, $\beta$-oxybutyrate dehydrogenase, nonspecific esterase, the increased amount of protein and glycogen, the expression of Fc $\gamma$ receptors, and oxygen metabolism (Shcherbakova et al., 1981; Zemskov et al., 1985b; Zemskov V. M. and Zemskov A. M., 1992). An early activation of lactate dehydrogenase responsible for intensification of glycolytic processes, malate dehydrogenase which serves as an energy supply marker, and glutamate dehydrogenase which reflects the intensity of amino acid metabolism was observed in thymus cells under the action of yeast RNA. The spectrum of targets of these biologically active compounds includes formation and interaction of cyclic nucleotides CAMP/cGMP which are known to regulate mitotic processes in various cells which act as second messengers that mediate the effects of corticosteroids, other hormones, enzyme systems of various cells, etc.

f) Free-radical Lipid and Protein Oxidation and the Antioxidant System as Bio regulators

\section{i. Free-radical Oxidation Processes}

These processes are central to cell metabolism. They act as a source of energy required for vital functions of a cell and the organism as a whole, "prepare" organic substances for cell structures, and participate in the metabolism of carbohydrates, lipids, proteins, and nucleic acids. Reactions of mitochondrial and microsomal oxidation resulting from incomplete reduction of oxygen to water produce active forms, such as singlet oxygen, superoxide anion radical, hydroxyl radical, hydroperoxyl radical, and hydrogen peroxide, which form peroxide compounds in the presence of mixed-valent metal ions.

The high biological activity of free-radical oxidation products determines two opposite types of their action in the body. The primary products which are normally observed in relatively low concentrations have a positive effect, i.e. reversible transformations of fatty acid residues of membrane phospholipids of various cells, with a positive change in the functional state of biomembranes and their enzymes. The secondary products of free-radical oxidation have a damaging effect on the structural and functional state of biomembranes, biomolecules and, first of all, proteins, including those of the immune system. Diene conjugates, ketodiens, malondialdehyde, fluorescent Schiff bases, CO-terminal residues of amino acids, bityrosine cross-links, etc., act as free-radical oxidation markers (Luzkiy et al., 2016).

\section{ii. Antioxidant Defense System}

Accumulation of toxic immunosuppressive molecules in the body requires the presence of a balanced antioxidant defense system consisting of enzymatic (superoxide dismutase, catalase, glutathione peroxidase, glutathione reductase, ceruloplasmin) and nonenzymatic (peroxidase resistance of red blood cells, $\alpha$-tocopherol, antiradical activity of lipids, total, nonprotein and protein thiols, total antioxidative activity) mechanisms. The balanced interaction of enzymatic and nonenzymatic components of an endogenous antioxidant defense system ensures stable free-radical chain reactions and the maintenance of concentrations of reactive oxygen species, free radicals, and molecular products of free-radical lipid and protein oxidation at a stationary level.

Free-radical lipid and protein oxidation reactions that are not balanced by the antioxidant defense result in oxidative stress in immune cells, which has a negative impact on the membranes and receptors of immune competent cells, which interferes with the cooperation processes, immune response development, and other phenomena, including those observed during infection processes (Zemskov et al., 2016b).

\section{g) Correlations between Immune-metabolic Parameters}

The presence of mathematically significant associations confirms involvement of the components discussed above in the pathogenesis of infections. It has been established that parameters of immunelaboratory status form a matrix of integrative associations of some kind, including intrasystem (between immune parameters), intersystem (between immune and routine hematological parameters), and non-system (between immune and metabolic parameters) associations.

i. Intra- and Intersystem Associations between Immune-metabolic Parameters

\section{a. Healthy Individuals}

In healthy individuals, T-cells were mathematically positively associated with total lymphocytes and monocytes, T-helpers-with total Tcells, lymphocytes, and monocytes, and B-cells had the same set of correlations with T-cells, T-helpers, cytotoxic T lymphocytes, and monocytes. Total T-cells, T-helpers, and B-cells had a positive correlation with segmented cells, and the phagocytic number had a negative correlation with stab cells. Total T-cells had a negative correlation with basophiles, and T-helpers-with eosinophils, while the phagocytic number had a positive correlation with the same cells. 
b. Purulent Soft Tissue Infection

In this pathology, there was a positive correlation between cytotoxic $T$ lymphocytes and white blood cells, a negative correlation between eosinophils and lymphocytes, and a positive correlation between Bcells and IgG and T-helpers.

\section{c. Acute Adnexitis}

In cases of acute adnexitis, IgM, IgA, and circulating immune complexes were characterized by a positive correlation with the white blood cell count, lymphocyte count, the level of proinflammatory TNF- $\alpha$, and had a negative correlation with the level of natural killer cells, T-helpers, IgG, and phagocytic index. In patients with urogenital chlamydiosis, IgM, oxygen activity of phagocytes determined by nitrobluetetrazolium reduction and total T-cells formed positive correlations with B-cells, T-helpers, phagocytes, lymphocytes, and natural killer cells.

\section{d. HbsAg Carriers}

In HbsAg carriers, lymphocytes and stab cells had a positive correlation with T-cells and IgM, while segmented cells and eosinophils had a negative correlation with T-cells and IgG, and T-cells were characterized by a positive correlation with null cells. There was a positive correlation between the monocyte count and the level of T-helpers, cytotoxic T lymphocytes, and the phagocytic number (Zemskov et al., 2007).

ii. Non-system Associations between Immunemetabolic Parameters

\section{a. Healthy Individuals}

In healthy individuals, the level of total T-cells had positive correlations with the «thymol test», blood amylase, cholesterol, prothrombin, antioxidative activity of plasma, and superoxide dismutase activity, and a negative correlation with alanine aminotransferase. Cytotoxic T lymphocytes had a correlation with total and indirect bilirubin, «thymol test», blood amylase, glucose, and alanine aminotransferase. T-helpers had a mathematical association with the «thymol test», prothrombin, and the antioxidative activity of plasma and superoxide dismutase. All three types of cells were characterized by a reliable negative correlation with alanine aminotransferase. Killer lymphocytes were dependent on the antioxidative activity of plasma and superoxide dismutase. The levels of total and direct bilirubin, prothrombin, the antioxidative activity of plasma and superoxide dismutase had a positive correlation with the B-cell count.

\section{b. Serous Meningitis}

In children with serous meningitis, there was a positive correlation between T-cells and their regulatory subpopulations, phagocytic index and alanine aminotransferase, B-cells and albumin, as well as phagocytic number and $\alpha-1$ and $\beta$-protein fractions.

\section{c. Purulent Meningitis}

In cases of a more severe purulent meningitis, cytotoxic $T$ lymphocytes had a positive correlation with cholesterol and $\beta$-lipoproteins, while T- and B-cells and IgM had a positive correlation with blood protein fractions.

\section{d. Nonspecific Pulmonary Inflammatory Diseases}

Nonspecific pulmonary inflammatory diseases in adults are characterized by inverse relationship between the level of ribonucleotides and the null cell count, and direct relationship with T-cells and IgA. T-cells, cytotoxic $T$ lymphocytes, and T-helpers had a negative correlation with lipid peroxidation products, i.e. malondialdehyde and glutathione peroxidase, circulating immune complexes and average weight molecules. Thus, the studies have shown that infectious diseases of the bronchopulmonary system are accompanied by an activation of lipid peroxidation processes, which results in an increase of cholesterol and $\beta$-lipoprotein level, along with a decrease in antioxidant defense activity, accumulation of biogenic amines, an increase in the level of polyunsaturated fatty acids in the bronchoalveolar contents, and a decrease in the content of more saturated acids. These changes are observed against the development of disnucleotidosis in patients, i.e. a violation of protein synthesis processes implemented according to the "DNA $\rightarrow$ RNA $\rightarrow$ protein" scheme. On the one hand, it causes inhibition of immune responses, especially cellmediated ones, and imbalance of regulatory lymphoid cell subpopulations; on the other hand, it promotes the development of allergic reaction; on the third hand, it results in the functional and destructive changes in cells of the bronchopulmonary system and other systems of the body; and on the fourth hand, it causes disorders which are closely associated with immune and neuroendocrine homeostasis regulation (Zemskov et al., 2016c).

\section{References Références Referencias}

1. Vorobyev, A. A., Krivoshein, Y. S. and Shirobokov, V. P. 2010. Medical and Sanitary Microbiology. Manual. $4^{\text {th }}$ edition. Moscow. Publishing Center: I. M. Sechenov First Moscow State Medical University. 464p. Russia.

2. Pokrovsky, V. I., Pak, S. G. and Briko, N. I. 2013. Infectious diseases and epidemiology. Textbook. $3^{\text {rd }}$ revised edition. Moscow. 1008p. Russia.

3. Zemskov, A. M. and Zemskov, V. M. 2016. An Integral Concept of Regulating Immune Homeostasis. J Clin Exp Path., 6(2): 1 - 5.

4. Pokrovsky V. I. 2012. National conception of prevention of infections associated with first aid rendering and informational material based on its provisions. Nizhny Novgorod. Publishing House: Remedium Privolzhye. 84 p. Russia. 
5. Zemskov, A. M., Esaulenko, I. E., Chereshnev, V. A., Zemskov, V. M., Suchkov S. V., Kozlov, V. A., Karaulov A. V., Konoplya A. I., Bolotskih V. I. and Popov V. I. et al. 2016a. Immunology. Electronic Textbook for Students of Medical Higher Education Institution and Faculties with Applications. Moscow. Publishing House: GEOTAR-media, 265 author's pages. Russia. http://www.studentlibrary.ru/ru/book/ 01-GEO-2016 01.html.

6. Karaulov, A. V., Aleshkin, V. A., Afanasiev, S. S. and Nesvizhsky, Y. V.(Ed.). 2015. New in the physiology of mucosal immunity. Moscow. Publishing House: I. M. Sechenov First Moscow State Medical University.167p. Russia.

7. Zemskov, A. M., Zemskova, V. A. and Voronzov, Z. A. 2016b. An Integral Concept of Regulating Immunogenesis. Academic Publishing Deuchland: LAP LAMBERT. 223p. Germany.

8. Zemskov, A. M., Zemskov, V. M. and Chereshnev, V. A. (Ed.). 2013. Encyclopedia of immunology in 5 volumes. Moscow. Publishing House: TriadaX.1962p. Russia.

9. Braun, W. 1968. Bacterial genetics. Moscow. Publishing House: Nauka.446p. Russia.

10. Zemskov, V. M., Lidak, M. Yu., Zemskov A. M. and Mikstays U. Ya. 1985 a. Low Molecular weight RNA. Obtaining, hydrolysis and use in medicine. Riga. Publishing House: Zinatne.191p. Russia.

11. Zemskov, V. M. and Zemskov A. M. 1992. Immuno modulating effects of a low molecular weight RNA. Soviet Medical Reviews/Section D. Immunology Reviews. Ed. by R.V. Petrov. Harwood Academic Publishers, Churchill-London-Paris-New YorkMelbourne. v. 3 (3): 113p. England.

12. Shcherbakova, E. G., Zemskov, V. M., Sobolev, V. R., Rastunova, G. A. and Barsukov A. A. 1981. Histochemical study of peritoneal macrophages activated by sodium nucleinate. Antibiotics. 3: 119-123. Russia.

13. Zemskov, V. M., Rodionov, S. V., Pantin, V. I., Khramtsov, A. V. and Mikstays, U. Ya. 1985b. Qualitative biochemical analysis of macrophages of mice stimulated by sodium nucleinate. Immunology. 6: 55-58. Russia.

14. Lutskiy, M., Zemskov, A. And Choporov, O. 2016. Oxidative stress in immune cells in cerebrovascular diseases. Academic Publishing Deutschland: LAP LAMBERT.223p. Germany.

15. Zemskov, A. M., Zemskov, V. M., Korotkikh, I. N., Zemskov, M. A. and Korotkikh, N. N. 2007. Immune disorders and their correction in pyoinflammatory processes. Moscow. Publishing House: TriadaX.159p. Russia.

16. Zemskov, A., Zemskov, M. and Zoloedov, V. 2016c. Immunology of chronic obstructive pulmonary diseases. Academic Publishing Deutschland: LAP LAMBERT. 676p. Germany. 